\title{
FOREST RESOURCES STUDY IN MONGOLIA USING ADVANCED SPATIAL TECHNOLOGIES
}

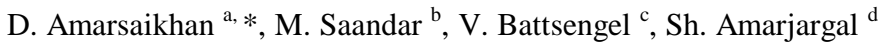 \\ a Institute of Informatics and RS, MAS, Ulaanbaatar-51, Mongolia - amar64@arvis.ac.mn \\ ${ }^{\mathrm{b}}$ MonMap Engineering Services Co., Ulaanbaatar, Mongolia - msaandar@mongol.net \\ ${ }^{c}$ School of Geography and Geology, NUM, Ulaanbaatar, Mongolia - battsengel@ num.edu.mn \\ ${ }^{\mathrm{d}}$ Research Centre of Astronomy and Geophysics, MAS, Ulaanbaatar, Mongolia - amarshrgl@yahoo.com
}

Commission VII, WG VII/5

KEY WORDS: Optical, Microwave, Forest mapping, Refined maximum likelihood classification

\begin{abstract}
:
The aim of this study is to conduct a forest resources study using optical and synthetic aperture radar (SAR) satellite images. For this purpose, a forest-dominated site around the Lake Khuvsgul located in northern Mongolia is selected. As remote sensing (RS) data sources, panchromatic and multispectral Landsat 7 images as well as ALOS PALSAR L-band HH polarization data are used. To produce a reliable land cover map from the multisensor images, a novel refined maximum likelihood classification based on the spectral and spatial thresholds are applied and for the accuracy assessment an overall accuracy is used. Overall, the research demonstrates that advanced spatial technologies based on optical and microwave RS are reliable tools for different forest studies.
\end{abstract}

\section{INTRODUCTION}

Forest is a very important natural resource that plays a significant role in keeping an environmental stability, ecological balance, environmental conservation, food security and sustainable development in both developed and developing countries. In recent years, deforestation and forest land degradation have become the main concern for forest specialists and ecologists as well as policy and decision-makers dealing with the environment. It has been found that much of the existing forests have been destroyed, mainly by shifting cultivation, timber preparation, legal and illegal logging, forest fires and increased number of people involved in agricultural activities Amarsaikhan et al. 2011). To protect and conserve the deteriorating forest, it is very important to introduce a sustainable forest management policy.

The concept of sustainable forest management should involve clear definition of the desired future condition of a forest, evaluation of the current management practices and thoroughly defined objectives to achieve a desired future condition. Achieving sustainable forest management should require effective measuring and monitoring activities which can provide reliable forest information to support policy and decision-making related to sustainable development (Haase and Camphausen, 2007). It is unambiguous that accurate and real-time forest related spatial information along with its attributes is the key for successful planning and management. In spatial context, such information might be collected from many different sources. However, the most reliable source that could provide real-time information for the accurate analysis could be RS (Amarsaikhan, 2011).
Traditionally, multispectral RS images have been widely used for forest monitoring and management. Since the end of the last century, single polarization SAR data sets have been increasingly accessible for the forest specialists. As the present space science and technology are so advanced, very high resolution multichannel optical and polarimetric SAR images are available for different forest studies. The combined application of optical and radar data sets can provide unique information for forest planning and management, because passive sensor images will represent spectral variations of the top layer of the forest classes, whereas microwave data with its penetrating capabilities can provide some additional information about forest canopy (Amarsaikhan et al. 2004, Amarsaikhan et al. 2011). Moreover, it is clear that the integrated use of the optical and microwave data sets should improve an accuracy in a forest mapping, because as the images are acquired in different portions of electro-magnetic spectrum, could positively influence a decision-making in overlapping boundaries (Amarsaikhan et al. 2009).

The aim of this research is to conduct a forest resources study using optical and microwave RS along with a geographical information system (GIS). For this purpose, a test site located in northern Mongolia has been selected. As RS data sources, multitemporal Landsat ETM+ images and ALOS PALSAR Lband $\mathrm{HH}$ polarization data were used. To produce a land cover map from the multisensor images, a novel refined maximum likelihood classification based on the spectral and spatial thresholds has been applied and for the accuracy assessment an overall accuracy was used. The analysis was carried out using Erdas Imagine and ArcGIS systems installed in a PC environment.

\footnotetext{
* Corresponding author
} 


\section{THE STUDY AREA AND DATA SOURCES}

Mongolia has a relatively low forest cover, although, the country has a total land area of 156.5 million hectares. Forest reserve land comprises 18.3 million hectares with 12.9 million hectares of forest-covered area. This includes 10.5 million hectares of coniferous and hardwood forests. The forests are mainly located in the northern and central parts of the country, forming a transition zone between the Siberian boreal forest and the Central Asian steppe. Climate change, global warming and negative human activities are directly and negatively influencing the Mongolia's forest resource and its quality. As the forests in Mongolia are state-owned, the Ministry for Nature, Environment and Tourism takes the overall responsibility for forest resource management. The main objective of the forest management is to protect and develop the existing forests of Mongolia so that they make maximum contributions to soil and watershed protection, and conservation of existing ecosystems. In the meantime, the forests are expected to produce, on a sustainable basis, increased volumes of industrial wood, fuel wood and minor forest products for the needs of people (Ykhanbai 2010).

For a test site, a coniferous forest-dominated area around the Lake Khuvsgul located in northern Mongolia has been selected. The lake is considered as the second largest fresh water lake in Asia after the Lake Baikal with $100 \mathrm{~km}$ in length, $35 \mathrm{~km}$ in width, and over $265 \mathrm{~m}$ in depth. The lake lies at $1645 \mathrm{~m}$ above sea level where mountains on the western shore rise up to $2961 \mathrm{~m}$ and the mountains on the northern shore even up to $3491 \mathrm{~m}$.The area surrounding the Lake Khuvsgul represents a forest ecosystem and is characterized by such main classes as coniferous forest, deciduous forest, grassland, light soil, dark soil and water. The annual precipitation in the region is about $350-400 \mathrm{~mm}$ and it makes the area as the most humid region in the country.

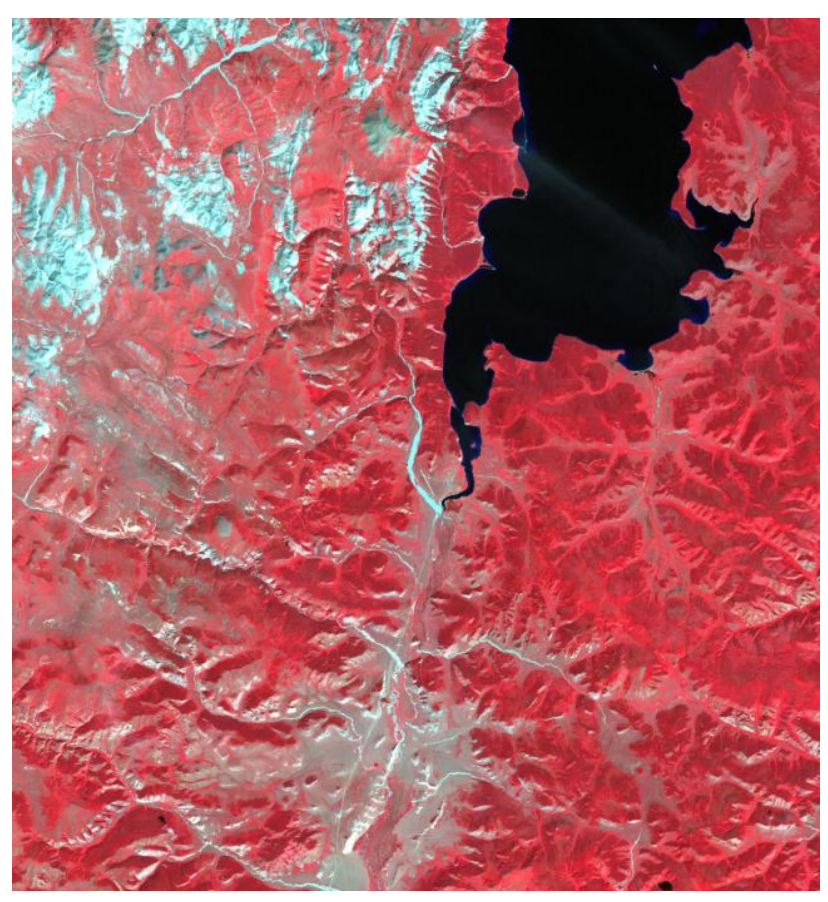

Figure 1. Landsat ETM+ image of the test area $(\mathrm{R}=\mathrm{B} 4, \mathrm{G}=\mathrm{B} 3, \mathrm{~B}=\mathrm{B} 2)$.
As data sources, multispectral Landsat ETM+ data of 12 August 2007 with a spatial resolution of $28 \mathrm{~m}$, a panchromatic Landsat ETM+ image of 28 August September 2007 with a spatial resolution of $15 \mathrm{~m}$ and ALOS PALSAR L-band $\mathrm{HH}$ polarization image of 17 August 2007 with a spatial resolution of $25 \mathrm{~m}$, a topographic map of 1986, scale 1:100,000 and a forest taxonomy map of 1984, scale 1: 500,000 have been used. The selected test site in the Landsat ETM+ image frame is shown in figure 1.

\section{GEOMETRIC CORRECTION OF THE IMAGES AND SPECKLE SUPPRESSION OF THE SAR IMAGE}

Initially, the multispectral and panchromatic Landsat ETM+ images were geometrically corrected to a UTM map projection using a topographic map of the study area, scale 1:100,000. In general, the panchromatic image was used to improve textural variety of the forests especially in bordering areas with other classes. The ground control point (GCP)s have been selected on clearly delineated crossings of rivers and other clear sites. In total 15 points were selected. For the transformation, a second order transformation and nearest neighbour resampling approach have been applied and the related root mean square (RMS) errors were 0.96 pixel and 0.83 pixel, respectively. In order to geometrically correct the PALSAR image, 18 more regularly distributed GCPs were selected comparing the locations of the selected points with other information such as Landsat ETM+ image and the topographic map. Then, the image was georeferenced to a UTM map projection using the topographic map of the study area. For the actual transformation, a second order transformation and nearest neighbour resampling approach were applied and the related RMS error was 1.18 pixel.

As the microwave images have a granular appearance due to the speckle formed as a result of the coherent radiation used for radar systems; the reduction of the speckle is a very important step in further analysis. The analysis of the radar images must be based on the techniques that remove the speckle effects while considering the intrinsic texture of the image frame (Serkan et al. 2008, Amarsaikhan et al. 2012). In this study, four different speckle suppression techniques such as local region, lee-sigma, frost and gammamap filters (ERDAS 1999) of $3 \times 3$ and $5 \times 5$ sizes were applied to the PALSAR image and compared in terms of delineation of forest and other texture information. After visual inspection of each image, it was found that the $5 \times 5$ gammamap filter created the best image in terms of delineation of different features as well as preserving content of texture information. In the output image, speckle noise was reduced with very low degradation of the textural information. After the speckle suppression, the SAR image was added to the optical bands, thus forming multisource images.

\section{DERIVATION OF FEATURES AND STANDARD MAXIMUM LIKELIHOOD CLASSIFICATION}

Generally, it is desirable to add some orthogonal features to any classification process to increase its decision-making. In the present study, for this aim, texture features have been used. To derive the texture features from the combined Landsat and PALSAR images, contrast and dissimilarity measures (using an $11 \times 11$ window size) have been applied and the results were compared. The bases for these measures are the co-occurrence measures that use a grey-tone spatial dependence matrix to calculate texture values, and the matrix shows the number of occurrences of the relationship between a pixel and its 
specified neighbour (ENVI 1999). The contrast measure indicates how most elements do not lie on the main diagonal, whereas, the dissimilarity measure indicates how different the elements of the co-occurrence matrix are from each other (Lee et al. 2004). By applying these measures, initially 12 features have been derived, but after thorough checking of each individual feature only 2 features, including the result of the contrast measure applied to the green band and the result of the dissimilarity measure applied to the near infrared band of Landsat, were selected.

To define the sites for the training signature selection from the images, two to three areas of interest (AOI) representing the selected classes (i.e., coniferous forest, deciduous forest, grassland, light soil, dark soil and water) have been selected. As the data sources included both optical and SAR features, the fused images were very useful for the determination of the homogeneous AOI as well as for the initial intelligent guess of the training sites. The separability of the training signatures was firstly checked in feature space and then evaluated using transformed divergence (Mather and Koch 2010). After the investigation, the samples that demonstrated the greatest separability were chosen to form the final signatures. The final signatures included about 563-992 pixels. For the classification, the following feature combinations were used:

1. The original spectral bands of the multitemporal Landsat data.

2. PALSAR and original bands of the multitemporal Landsat data.

3. Multiple bands including the original PALSAR and Landsat images as well as two other derivative bands obtained from texture measures.

For the actual classification, a standard maximum likelihood classification has been used assuming that the training samples have the Gaussian distribution. The maximum likelihood classification is the most widely used statistical classification technique, because a pixel classified by this method has the maximum probability of correct assignment (Erbek et al. 2004).

To increase the reliability of the classification, to the initially classified images, a fuzzy convolution with a $3 \times 3$ size window was applied. The fuzzy convolution creates a thematic layer by calculating the total weighted inverse distance of all the classes in a determined window of pixels and assigning the centre pixel the class with the largest total inverse distance summed over the entire set of fuzzy classification layers, i.e. classes with a very small distance value will remain unchanged while the classes with higher distance values might change to a neighboring value if there are a sufficient number of neighboring pixels with class values and small corresponding distance values (ERDAS 1999). The visual inspection of the fuzzy convolved images indicated that there are some improvements on the borders of the neighboring classes that significantly influence the separation of the decision boundaries in multidimensional feature space. The final classified images are shown in figure $3(a-c)$. As seen from figure $3(a-c)$, the classification result of the Landsat image gives the worst result, because there are high overlaps among grassland and forest classes. However, these overlaps decrease on other images for the classification of which SAR and optical bands as well as other derivative features have been used.
For the accuracy assessment of the classification results, the overall performance has been used. This approach creates a confusion matrix in which reference pixels are compared with the classified pixels and as a result an accuracy report is generated indicating the percentages of the overall accuracy (Richards and Jia, 1999). As ground truth information, different AOIs containing 5,751 purest pixels have been selected. AOIs were selected on a principle that more pixels to be selected for the evaluation of the larger classes such as grassland and coniferous forest than the smaller classes such as deciduous forest and dark soil. The overall classification accuracies for the selected classes were $74.35 \%, 79.18 \%$ and $83.09 \%$ for the original Landsat bands, the combined features and multiple bands, respectively.

As could be seen from the overall classification results, although the combined use of optical and SAR data sets produced a better result than the single source image, it is still very difficult to obtain a reliable forest map by the use of the standard technique, specifically on decision boundaries of the statistically overlapping classes.

\section{THE REFINED MAXIMUM LIKELIHOOD CLASSIFICATION}

For several decades, single-source multispectral data sets have been effectively used for a land cover mapping. Unlike singlesource data, multisource data sets have proved to offer better potential for discriminating between different land cover types (Amarsaikhan et al. 2012). Generally, it is very important to design a suitable image processing procedure in order to successfully classify any RS data into a number of class labels. The effective use of different features derived from different sources and the selection of a reliable classification technique can be a key significance for the improvement of classification accuracy (Lu and Weng, 2007). In this study, for the classification of land cover types, a refined statistical maximum likelihood classification algorithm has been constructed. As the features, multiple bands that include the original Landsat and PALSAR images as well as two other derivative bands obtained from texture measures have been used.

Unlike the traditional method, the constructed classification algorithm uses spectral and spatial thresholds defined from the contextual knowledge. The contextual knowledge was defined on the basis of the spectral variations of the land surface features on the fused images as well as the texture information delineated on the dissimilarity image. For determination of the spectral thresholds, maximum and minimum values of the selected training signatures and the pixels falling within 1.0 standard deviation were compared, and the latter was selected. It is clear that a spectral classifier will be ineffective if applied to the statistically overlapping classes, because they have very similar spectral characteristics. For such spectrally mixed classes, classification accuracies should be improved if the spatial properties of the classes of objects could be incorporated into the classification criteria. The idea of the spatial threshold is that it uses a polygon boundary to separate the overlapping classes and only the pixels falling within the threshold boundary are used for the classification. In that case, the likelihood of the pixels to be correctly classified will significantly increase, because the pixels belonging to the class that overlaps with the class to be classified using the threshold 
a)

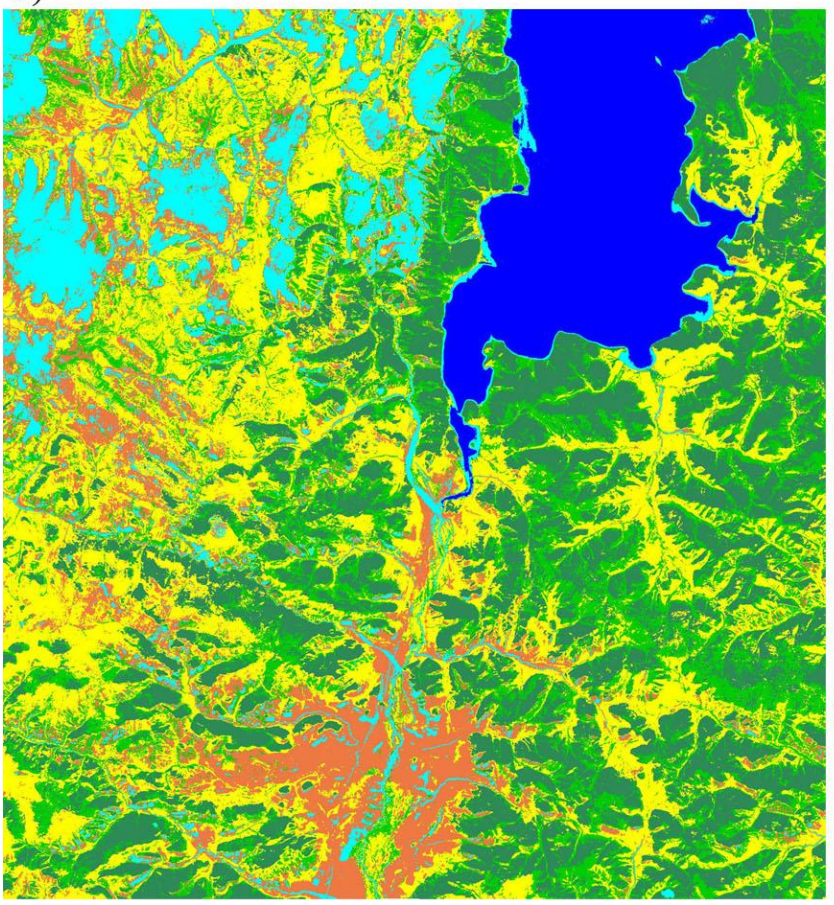

c)

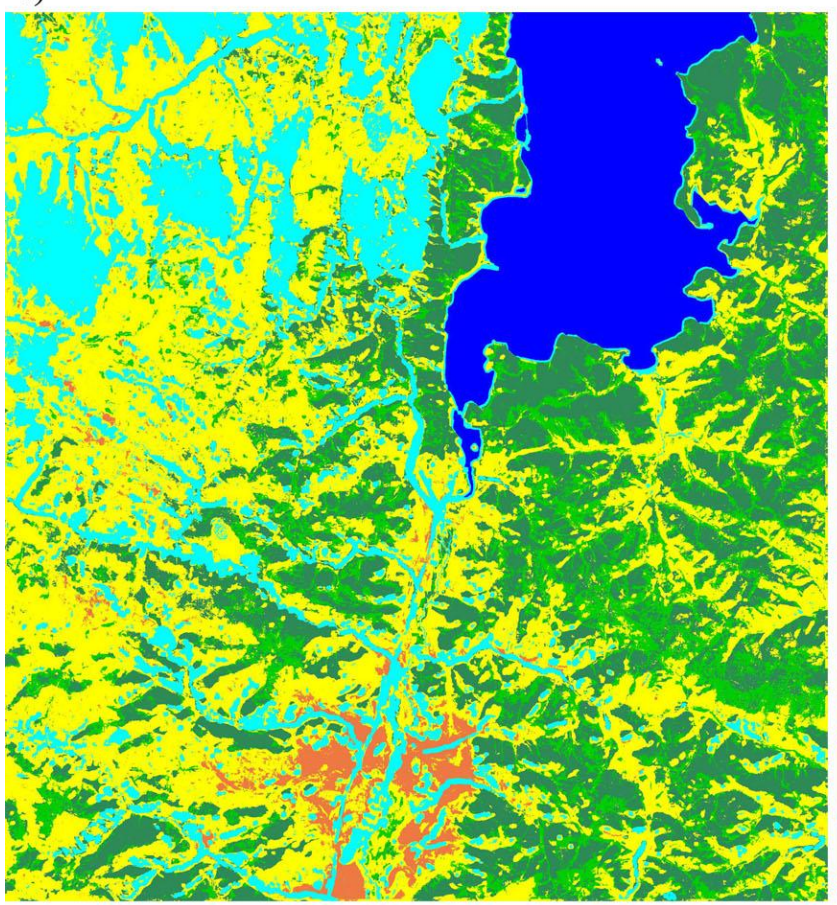

Coniferous

Deciduous b)

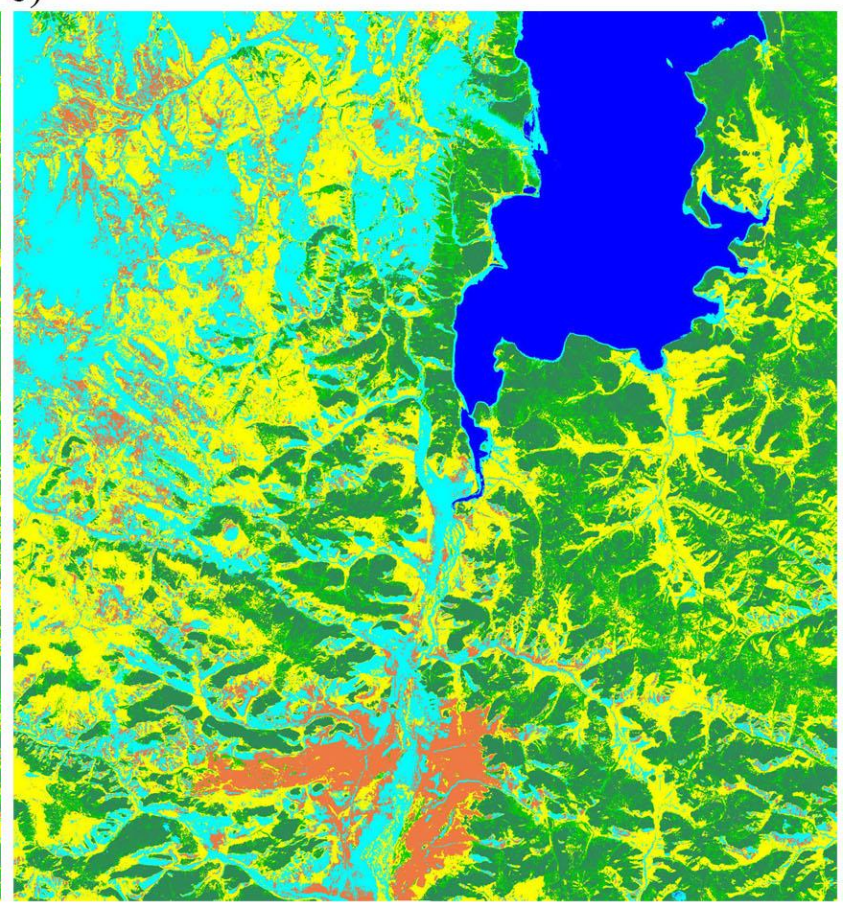

d)

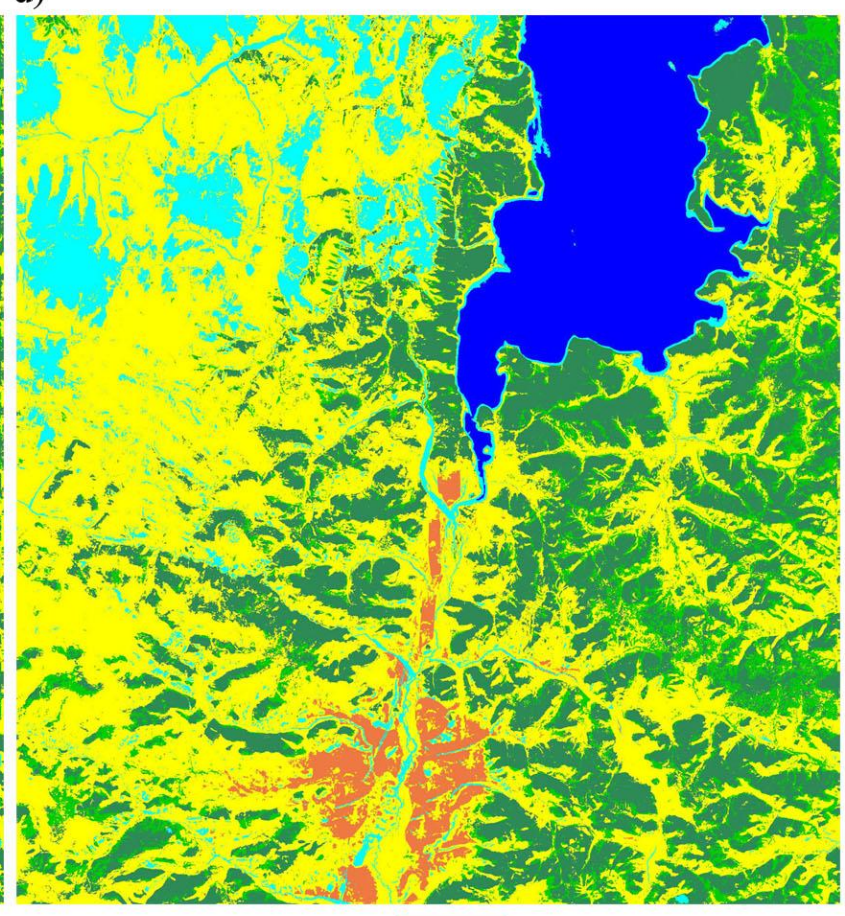

\section{Light soil \\ Dark soil}

Figure 2. Comparison of the classification results for the selected classes:

(a) Classified image using original spectral bands of the multitemporal Landsat data,

(b) Classified image using PALSAR and original bands of the multitemporal Landsat data,

(c) Classified image using optical, SAR and two other derivative bands,

(d) Classified image using the refined classification. 
boundary are temporarily excluded from the decision making process. In such a way, the image can be classified several times using different threshold boundaries and the results can be merged (Amarsaikhan et al. 2010).

The result of the classification using the refined method is shown in figure $2 \mathrm{~d}$. For the accuracy assessment of the classification result, the overall performance has been used, taking the same number of sample points as in the previous classifications. The confusion matrix produced for the refined classification method showed overall accuracy of $90.78 \%$. As could be seen from figure $2 \mathrm{~d}$, the result of the classification using the refined classification is better than the results of the standard method. A general diagram of the refined classification method is shown is figure 3 .

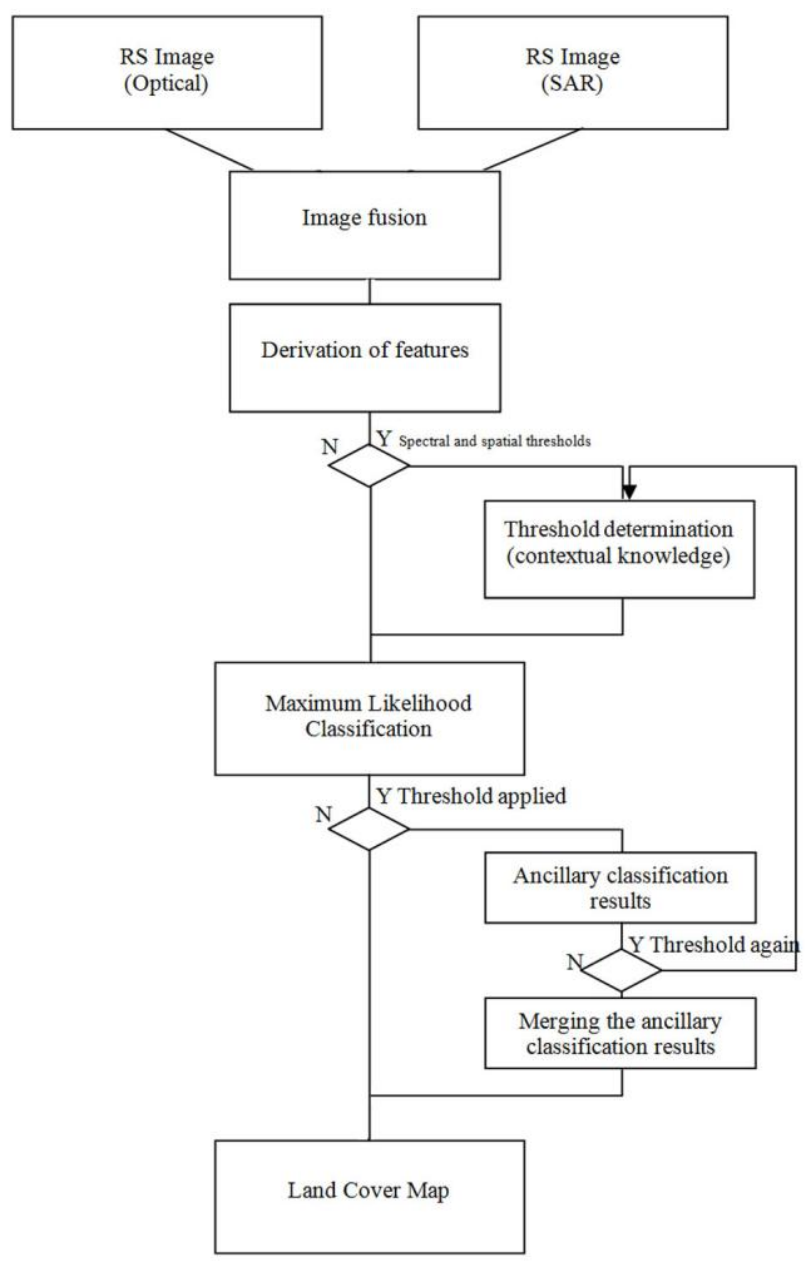

Figure 3. A general diagram of the refined classification.

To compare the final result with the existing information, a GIS layer was created using a forest map of 1984 and for its digitizing ArcGIS system was used. It is the only forest map available in the region and a digitized map is shown in figure 4. The initial aim of the study was to compare the forest changes occurred between these two periods However, the existing forest map was not reliable, because ground truth information and contextual knowledge indicated that it is not accurate at all. This is a common problem in many of the developing countries, where the old maps need to be updated through processing of satellite images. In the current study, as the overall classification accuracy of the classified multisource images is more than $90 \%$, the result can be directly used to update the existing forest layer and used for planning and management.

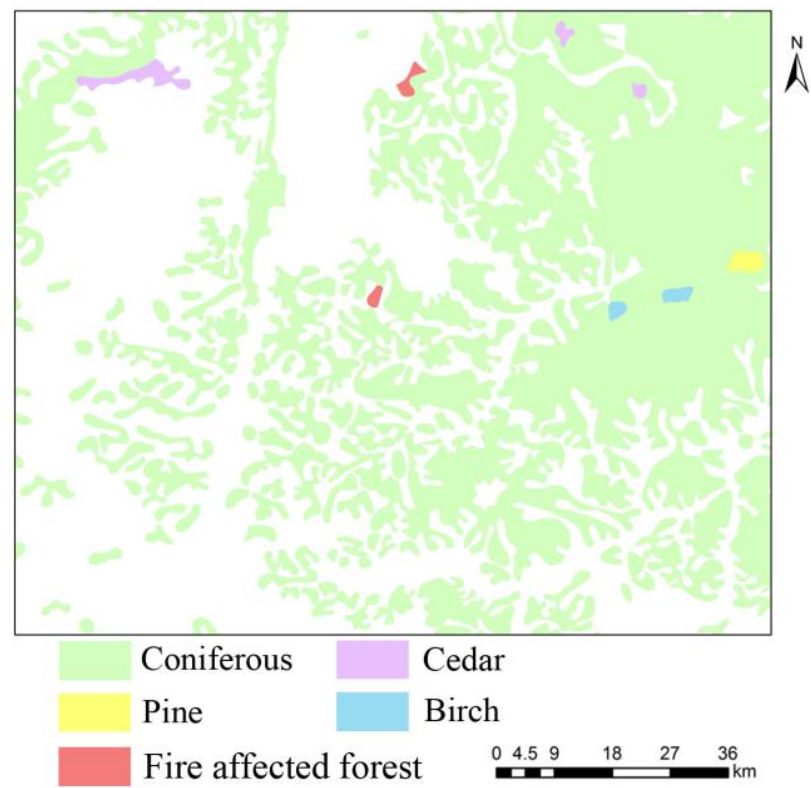

Figure 4. A digitized forest map of the test region.

\section{CONCLUSIONS}

The aim of this research was to conduct a forest resources study in northern Mongolia using advanced spatial technologies. As data sources, panchromatic and multispectral Landsat 7 images, ALOS PALSAR L-band HH polarization data, a topographic map, and a forest taxonomy map were used. To produce a reliable land cover map from the multisensor images, a novel refined maximum likelihood classification based on the spectral and spatial thresholds defined from the contextual knowledge, was constructed. The contextual knowledge was defined on the basis of the spectral variations of the land surface features on the fused images as well as the texture information delineated on the dissimilarity image. For determination of the spectral thresholds, the pixels falling within 1.0 standard deviation were used. The result of the constructed method was better than the results of the traditional method and it could be used to update a forest layer within a GIS. Overall, the study demonstrated that advanced spatial technologies based on optical and microwave RS are reliable tools for forest planning and management.

\section{REFERENCES}

Amarsaikhan, D., Ganzorig, M., Batbayar, G., Narangerel, D. and Tumentsetseg, Sh., 2004. An integrated approach of optical and SAR images for forest change study. Asian Journal of Geoinformatics, 4(3), pp. 27-33.

Amarsaikhan, D., Bolorchuluun, Ch., Narangerel, Z. and Gantuya, R., 2009. Integration of RS and GIS for forest 
monitoring in Mongolia. In: CD-ROM Proceedings of the Asian Conference on RS, Beijing, China, October 2009.

Amarsaikhan, D., Blotevogel, H.H., van Genderen, J.L., Ganzorig, M., Gantuya, R. and Nergui, B., 2010. Fusing high resolution TerraSAR and Quickbird images for urban land cover study in Mongolia. International Journal of Image and Data Fusion, 1(1), pp. 83-97.

Amarsaikhan, D., 2011. Applications of advanced technology for combating land degradation and desertification in Mongolia. In: Proceedings of the Council Asia Conference, Ulaanbaatar, Mongolia, July 2011. pp. 39-50.

Amarsaikhan, D., Battsengel, V., Amarjargal, Sh., Egshiglen, E., Ganzorig, M. and Enkhjargal, D., 2011. Applications of optical and microwave RS for forest mapping in Mongolia. In: CD-ROM Proceedings of the ACRS, Taipei, Taiwan, October 2011.

Amarsaikhan, D., Saandar, M., Ganzorig, M., Blotevogel, H.H. and Enkhjargal, D., 2012. Comparison of multisource image fusion methods and land cover classification. International Journal of Remote Sensing, 33(8), pp. 2532-2550.

ENVI, 1999, User's Guide, Research Systems.

Erbek, F.S., Zkan, C.O. and Taberner, M., 2004. Comparison of maximum likelihood classification method with supervised artificial neural network algorithms for land use activities. International Journal of Remote Sensing, 25(9), pp. 17331748 .

ERDAS, 1999. Field guide. $5^{\text {th }}$ Ed. ERDAS, Inc. Atlanta, Georgia, USA.

Haase, G. and Camphausen, A., 2007. Best Practices for Sustainable Forest Management in Southeast Asia, Deutsche Gesellschaft fur Technische Zusammenarbeit GmbH, Jakarta, Indonesia, pp. 494.

Lee, K., Jeon, S.H. and Kwon, B.D., 2004. Urban feature characterization using high-resolution satellite imagery: texture analysis approach, MAPASIA 2004.

Lu, D. and Weng, Q., 2007. A survey of image classification methods and techniques for improving classification performance. International Journal of Remote Sensing, 28(5), pp. $823-870$.

Mather, P.M. and Koch, M., 2010. Computer Processing of Remotely-Sensed Images: An Introduction, Fourth Edition, (Wiley, John \& Sons).

Richards, J.A., and Jia, X., 1999. Remote Sensing Digital Image Analysis-An Introduction, Third Edition, (Berlin: Springer-Verlag).

Serkan, M., Musaoglu, N., Kirkici, H. and Ormeci, C., 2008, Edge and fine detail preservation in SAR images through speckle reduction with an adaptive mean filter. International Journal of Remote Sensing, 29(23), pp. $6727-6738$.
Ykhanbai, H., 2010, Mongolia Forestry Outlook Study, FAO of the UN Regional Office for Asia and the Pacific, Working Paper No. APFSOS II/ WP/ 2009/ 21, Bangkok, pp. 49. 